\title{
ALGEBRAIC SOLUTIONS OF THE LAMÉ EQUATION
}

\author{
FRITS BEUKERS \\ Department of Mathematics, University of Utrecht \\ P.O. Box 80.010, 3508 TA Utrecht, the Netherlands \\ E-mail: beukers@math.uu.nl
}

\begin{abstract}
In this paper we give a summary of joint work with Alexa van der Waall concerning Lamé equations having finite monodromy. This research is the subject of van der Waall's Ph. D. thesis $[\mathrm{W}]$.
\end{abstract}

1. Second order equations with finite monodromy. Consider the set of second order linear Fuchsian differential equations of the form

$$
L y=0, \quad L \in \mathbb{C}(z)\left[\frac{d}{d z}\right]
$$

having finite monodromy group. Denote this set by $\mathcal{A}$. As is well-known, $\mathcal{A}$ is precisely the set of second order equations over $\mathbb{C}(z)$ whose solution set consists of functions algebraic over $\mathbb{C}(z)$. By abuse of language we call the elements from $\mathcal{A}$ algebraic differential equations.

Consider an equation $L y=0$ from the set $\mathcal{A}$. At every point of $a \in \mathbb{P}^{1}$ the equation $L y=0$ has two local exponents $\rho_{1}, \rho_{2}$. We call $\left|\rho_{1}-\rho_{2}\right|$ the local exponent difference at $a$. At every non-singular point the local exponent difference is 1 . Suppose conversely that the local exponent difference of $L y=0$ at $a$ equals 1 . Since $L y=0$ belongs to $\mathcal{A}$ there are no local logarithmic solutions. Denote the local solutions at $a$ by $(z-a)^{\rho} f_{1}(z)$ and $(z-a)^{\rho+1} f_{2}(z)$, where $f_{1}, f_{2}$ are locally biholomorphic at $a$. Then the differential equation $(z-a)^{-\rho} L\left((z-a)^{\rho} y\right)=0$ has the solutions $f_{1},(z-a) f_{2}$ and $z=a$ is a non-singular point of the new differential equation.

An equation from $\mathcal{A}$ is called pure if the only integral exponent difference that is allowed to occur is 1 . In particular, apparent singularities are forbidden with such equations. Denote the subset of pure equations by $\mathcal{A}_{0}$. The set of pure equations is stable under the substitution $L \rightarrow A(z) R(z)^{-\rho} L \circ R(z)^{\rho}$ for any $A(z), R(z) \in \mathbb{C}(z)$ and $\rho \in \mathbb{Q}$. It is also stable under automorphisms of $\mathbb{P}^{1}$, that is, replacing $z$ by $\frac{a z+b}{c z+d}$ for any $\left(\begin{array}{ll}a & b \\ c & d\end{array}\right) \in G L(2, \mathbb{C})$.

2000 Mathematics Subject Classification: Primary 34A30; Secondary 34M50, 34M35.

The paper is in final form and no version of it will be published elsewhere. 
These two operations give an equivalence relation in $\mathcal{A}_{0}$. Denote this equivalence relation by $\sim$. We have the following Theorem.

Theorem 1.1. Let notations be as above. Then $\mathcal{A}_{0} / \sim$ is a countable set.

A proof of this Theorem is given in the last section. As a consequence of this Theorem one can start an enumeration of the set $\mathcal{A}_{0}$. We perform this enumeration using an increasing number of singular points of the differential equation. Let us start with Fuchsian equations having two singularities, which we may assume to be $0, \infty$. Such an equation is of the form $z^{2} \frac{d^{2} y}{d z^{2}}+a z \frac{d y}{d z}+b y=0$. It has a basis of solutions of the form $z^{\rho_{1}}, z^{\rho_{2}}$ where $\rho_{1}, \rho_{2}$ are zeros of $x^{2}+(a-1) x+b$. Algebraicity of the solutions is equivalent to $\rho_{1}, \rho_{2} \in \mathbb{Q}$. Hence $a, b \in \mathbb{Q}$.

The first interesting case is that of three singularities. By application of an equivalence transformation we can see to it that the singularities are $0,1, \infty$ and at 0,1 at least one local exponent is 0 . These properties characterise the Gaussian hypergeometric equation, having the famous hypergeometric series

$$
F(a, b, c \mid z)=\sum_{n=0}^{\infty} \frac{(a)_{n}(b)_{n}}{(c)_{n} n !} z^{n}
$$

as solution, where $(x)_{n}=x(x+1) \cdots(x+n-1)$ is the so-called Pochhammer symbol. The numbers $a, b, c$ are the parameters of the hypergeometric equation. In $1873 \mathrm{H}$. A. Schwarz [Schw], using ideas of Riemann, gave a complete list of all hypergeometric equations having an algebraic solution set.

The next step would be to study second order equations with four singularities. However, in this case we encounter a difficulty. In the previous cases the equation was determined by the location of the singularities and the local exponents. In other words, by local data. In the case of four singularities there is one parameter which is not determined by local data. This is called the accessory parameter. The dependence of the monodromy group on the accessory parameter is as yet little understood. It is possible however to find conditions on the accessory parameter for the solutions to be algebraic. In particular, we shall do this for the Lamé equation.

2. The Lamé equation. Let $n \in \mathbb{Q}, g_{2}, g_{3}, B \in \mathbb{C}$. The Lamé equation with these numbers as parameters is the equation given by

$$
p(z) \frac{d^{2} y}{d z^{2}}+\frac{1}{2} p^{\prime}(z) \frac{d y}{d z}-(n(n+1) z+B) y=0
$$

where $p(z)=4 z^{3}-g_{2} z-g_{3}$ and we assume that $p(z)$ has three distinct zeros $z_{1}, z_{2}, z_{3}$. This equation will be abbreviated by

$$
L_{n, B} y=0 .
$$

The local exponents are $0,1 / 2$ at the three finite singularities and $-n / 2,(n+1) / 2$ at $\infty$. Since the equation does not change under $n \rightarrow-1-n$ we shall assume $n \geq-1 / 2$. The number $B$ is the accessory parameter of the equation.

Consider a local set of solutions around a non-singular point and consider also the action of the monodromy group $M$ on this space. The local monodromies $\gamma_{i}$ at the 
finite singularities $z_{i}$ have eigenvalues \pm 1 and hence $\gamma_{1}^{2}=\gamma_{2}^{2}=\gamma_{3}^{2}=$ Id. Moreover, $\gamma_{1} \gamma_{2} \gamma_{3} \gamma_{\infty}=$ Id where $\gamma_{\infty}$ is the local monodromy at $\infty$. Moreover, $M$ is generated by the $\gamma_{i}$ and the $\gamma_{i}$ are reflections. So $M$ is a so-called reflection group.

There are two particular cases to be mentioned. The first one is $n+1 / 2 \in \mathbb{Z}$. Since $n+1 / 2$ is the local exponent difference at $\infty$, logarithmic solutions at $\infty$ may arise.

Theorem 2.1 (Brioschi-Halphén). Suppose $n+1 / 2 \in \mathbb{Z}_{\geq 0}$. Then there exists a polynomial $p_{n} \in \mathbb{Z}\left[g_{2} / 4, g_{3} / 4, B\right]$ of degree $n+1 / 2$ in $B$ such that $\bar{L}_{n, B} y=0$ has no logarithmic solutions at $\infty$ if and only if $p_{n}\left(g_{2}, g_{3}, B\right)=0$.

The polynomial $p_{n}$ is known as the Brioschi-Halphén determinant. In particular, if there are no logarithmic solutions, then $\gamma_{\infty}$ acts as a scalar. It is not hard to see that $\gamma_{i}^{2}=$ Id for $i=1,2,3$ and $\gamma_{1} \gamma_{2} \gamma_{3}$ scalar imply that $M$ modulo scalars equals Klein's four group $V_{4}$. In other words, if $p_{n}\left(g_{2}, g_{3}, B\right)=0$ then the monodromy group is finite. For example, when $n=3 / 2$ we have $p_{3 / 2}\left(g_{2}, g_{3}, B\right)=B^{2}-3 g_{2} / 4$. There are overcountably many $g_{2}, g_{3}, B$ satisfying $B^{2}-3 g_{2} / 4=0$. Notice also that our equation is not pure for such triples since the local exponent difference at $\infty$ is 2 . So we see that Theorem 1.1 cannot hold if we drop the purity condition.

The next case of interest is $n \in \mathbb{Z}$. Then $n+1 / 2$ is a half integer and $\gamma_{\infty}$ is also a reflection. So we now have

$$
\gamma_{1}^{2}=\gamma_{2}^{2}=\gamma_{3}^{2}=\gamma_{\infty}^{2}=\mathrm{Id}, \quad \gamma_{1} \gamma_{2} \gamma_{3} \gamma_{\infty}=\mathrm{Id}
$$

From these relations it follows easily that the subgroup $H$ generated by $\gamma_{1} \gamma_{2}$ and $\gamma_{2} \gamma_{3}$ is an abelian subgroup of $M$ of index 2 . We can now distinguish two cases.

1. $H$ contains an element with two distinct eigenvalues. Denote the corresponding eigenfunctions by $y_{1}, y_{2}$. With respect to this basis the group $H$ is a subgroup of

$$
\left\{\left(\begin{array}{cc}
\lambda & 0 \\
0 & \lambda^{-1}
\end{array}\right) \mid \lambda \in \mathbb{C}^{*}\right\}
$$

The monodromy group $M$ itself is then a subgroup of

$$
\left\{\left(\begin{array}{cc}
\lambda & 0 \\
0 & \lambda^{-1}
\end{array}\right),\left(\begin{array}{cc}
0 & \lambda \\
\lambda^{-1} & 0
\end{array}\right) \mid \lambda \in \mathbb{C}^{*}\right\} .
$$

2. All elements of $H$ have coinciding eigenvalues. Then, with respect to a suitable basis, $H$ is a subgroup of

$$
\left\{ \pm\left(\begin{array}{ll}
1 & \lambda \\
0 & 1
\end{array}\right) \mid \lambda \in \mathbb{C}\right\} .
$$

The monodromy group $M$ itself is then a subgroup of

$$
\left\{\left(\begin{array}{cc} 
\pm 1 & \lambda \\
0 & \pm 1
\end{array}\right) \mid \lambda \in \mathbb{C}\right\}
$$

and $M$ acts reducibly. The one-dimensional invariant subspace corresponds to the so-called Lamé solutions.

The following classical theorem characterises the occurrence of Lamé and Hermite solutions. 
Theorem 2.2 (Lamé). Suppose $n \in \mathbb{Z}_{\geq 0}$. Then there is a polynomial $p_{n} \in \mathbb{Z}\left[g_{2} / 4\right.$, $\left.g_{3} / 4, B\right]$ of degree $n$ in $B$ such that there exists a solution of the form

$$
\prod_{i=1}^{3}\left(z-z_{i}\right)^{\epsilon_{i}} Q(z)
$$

with $\epsilon_{i} \in\{0,1 / 2\}, Q(z) \in \mathbb{C}[z]$, if and only if $p_{n}\left(g_{2}, g_{3}, B\right)=0$.

Moreover, the case described in this Theorem is the only case in which the Lamé equation is reducible over $\mathbb{C}(z)$ (see $[\mathrm{W}]$ ).

3. Algebraic Lamé equations. In this section we suppose that the monodromy group $M$ of the Lamé equation is finite. The group $M$ is generated by the three local monodromy elements $\gamma_{1}, \gamma_{2}, \gamma_{3}$, each having eigenvalues \pm 1 . Through the classification of finite subgroups of $\operatorname{PGL}(2, \mathbb{C})$ we know that $M$ modulo scalars is either one of the following groups: the cyclic group $C_{n}$ of order $n$, the dihedral group $D_{n}$ of order $2 n$, the alternating groups $A_{4}, A_{5}$ and the permutation group $S_{4}$. Moreover, in each of these cases we can find an explicit description of the matrix group in $[\mathrm{K}]$. The following theorem is immediate.

Theorem 3.1 (Baldassarri). The group $M$ modulo scalars cannot be $A_{4}$.

This follows from the fact that the $\gamma_{i}$ still have order two if we consider them as elements of $P G L(2, \mathbb{C})$ and $A_{4}$ cannot be generated by elements of order two.

A more refined description of $M$ can be given when we use the classification of Shephard and Todd of finite complex reflection groups. A finite complex reflection group is a finite subgroup of $G L(m, \mathbb{C})$ which is generated by complex reflections. A complex reflection is a semi-simple element all of whose eigenvalues except one are equal to 1 . In the following theorem an element $g \in G L(m, \mathbb{C})$ acts on $\mathbb{C}\left[x_{1}, \ldots, x_{m}\right]$ via $\left(x_{1}, \ldots, x_{m}\right)^{t} \mapsto g\left(x_{1}, \ldots, x_{m}\right)^{t}$. The action of $g$ on a polynomial $P$ is denoted by $P^{g}$. We define

$$
\mathbb{C}\left[x_{1}, \ldots, x_{m}\right]^{G}:=\left\{P \in \mathbb{C}\left[x_{1}, \ldots, x_{m}\right] \mid P^{g}=P \text { for all } g \in G\right\} .
$$

Theorem 3.2 (Shephard-Todd). Let $G$ be a finite subgroup of $G L(m, \mathbb{C})$. Then $G$ is a finite complex reflection group if and only if $\mathbb{C}\left[x_{1}, \ldots, x_{m}\right]^{G}$ is a polynomial ring freely generated by $m$ elements $I_{1}, \ldots, I_{m}$.

Let $G$ be a finite complex reflection group and $I_{1}, \ldots, I_{m}$ be a set of generating invariants. We can assume them to be homogeneous polynomials. Denote the degree of $I_{i}$ by $d_{i}$ and suppose that $d_{1} \leq d_{2} \leq \cdots \leq d_{m}$. Then the $d_{i}$ are uniquely determined and they are called the degrees of $G$. In their paper [ST] Shephard and Todd also give a complete classification of all finite complex reflection groups. We can use their classification to list the possible finite monodromy groups $M$ that occur for the Lamé equation. In the case when $m=2$ we get, using the further restriction that $M$ is generated by order 2 reflections, the following list of possibilities.

$$
G(4,2,2), \quad G(N, N, 2)(N \geq 3), \quad G_{12}, \quad G_{13}, \quad G_{22} .
$$


Here $G(4,2,2)$ is the group of order 16 generated by

$$
\left(\begin{array}{cc}
i & 0 \\
0 & -i
\end{array}\right),\left(\begin{array}{cc}
-1 & 0 \\
0 & 1
\end{array}\right),\left(\begin{array}{ll}
0 & 1 \\
1 & 0
\end{array}\right) \text {. }
$$

Its quotient by scalars is Klein's four group $V_{4}$. The group $G(N, N, 2)$ is the dihedral group of order $2 N$ generated by

$$
\left(\begin{array}{cc}
\exp (2 \pi i / N) & 0 \\
0 & \exp (-2 \pi i / N)
\end{array}\right),\left(\begin{array}{ll}
0 & 1 \\
1 & 0
\end{array}\right) .
$$

The group $G_{12}$ is generated by

$$
\frac{1}{\sqrt{2}}\left(\begin{array}{cc}
0 & 1+i \\
1-i & 0
\end{array}\right), \frac{1}{\sqrt{2}}\left(\begin{array}{cc}
1 & 1 \\
1 & -1
\end{array}\right), \frac{1}{\sqrt{2}}\left(\begin{array}{cc}
1 & i \\
-i & -1
\end{array}\right) .
$$

The group $G_{13}$ is the group generated by the elements of $G_{12}$ together with $\left(\begin{array}{ll}i & 0 \\ 0 & i\end{array}\right)$. The groups $G_{12}, G_{13}$ modulo scalars are isomorphic to $S_{4}$. They will be called octahedral groups. Finally, the group $G_{22}$ is generated by

$$
\left(\begin{array}{cc}
i & 0 \\
0 & i
\end{array}\right), \frac{1}{\sqrt{5}}\left(\begin{array}{cc}
\zeta_{5}-\zeta_{5}^{4} & \zeta_{5}^{2}-\zeta_{5}^{3} \\
\zeta_{5}^{2}-\zeta_{5}^{3} & \zeta_{5}^{4}-\zeta_{5}
\end{array}\right), \frac{1}{\sqrt{5}}\left(\begin{array}{cc}
\zeta_{5}^{3}-\zeta_{5} & 1-\zeta_{5} \\
\zeta_{5}^{4}-1 & \zeta_{5}^{2}-\zeta_{5}^{4}
\end{array}\right) .
$$

Its quotient by scalars is $A_{5}$ and we call it the icosahedral group. We have the following Theorem.

Theorem 3.3 (Van der Waall). Suppose the Lamé equation $L_{n, B} y=0$ has finite monodromy group $M$. Then

1. $M=G(4,2,2) \Rightarrow n \in 1 / 2+\mathbb{Z}$

2. $M=G(N, N, 2) \Rightarrow n \in \mathbb{Z}$

3. $M=G_{12} \Rightarrow n \in \pm 1 / 4+\mathbb{Z}$

4. $M=G_{13} \Rightarrow n \in \pm 1 / 6+\mathbb{Z}$

5. $M=G_{22} \Rightarrow n \in \pm 1 / 10, \pm 3 / 10, \pm 1 / 6+\mathbb{Z}$

Moreover, in each of the cases 1,3,4,5 we can find a Lamé equation such that the group actually occurs together with the given residue class $n(\bmod \mathbb{Z})$. The group $G(4,4,2)$ never occurs. The group $G(N, N, 2)$ is known to occur for $N=3,5,6,7,8,9,10$.

A complete proof can be found in [W]. Partial results in this direction were obtained by Baldassarri [B] and Chiarellotto [C]. We expect that for every group $G(N, N, 2)$ with $N \geq 3, N \neq 4$, there is a Lamé equation having this monodromy group. In [C] there is a method to count the number of inequivalent Lamé equations with given dihedral monodromy group and thus confirm our expectation. Unfortunately Theorems 2.14, 2.15 in $[\mathrm{C}]$ only consider monodromy modulo scalars. For example, from $[\mathrm{C}]$ it follows that there are two distinct Lamé equations with $n=1$ and dihedral monodromy group of order 10. In $[\mathrm{W}]$ the author gets only one such equation, whereas the other intended solution has dihedral monodromy of order 20. Also it is stated in [C] that there is one case with $n=1$ and dihedral monodromy group of order 8 . This contradicts the above Theorem, which states that such groups cannot occur. We think that the group alluded to in $[\mathrm{C}]$ is in fact dihedral of order 16 . 
In $[\mathrm{B}]$ it is stated that the octahedral group cannot occur when $n \in 1 / 6+\mathbb{Z}$. However, this is due to an error since the Lamé equation with $g_{2}=1, g_{3}=0, B=0, n=1 / 6$ does have octahedral monodromy, as it is the rational pull-back of the hypergeometric equation $x(x-1) y^{\prime \prime}+(5 x / 4-3 / 4) y^{\prime}-\left(7 / 24^{2}\right) y=0$ by the substitution $x=z^{2}$. The latter hypergeometric equation has octahedral monodromy.

4. Enumeration of algebraic Lamé equations. For each choice of group $M$ and parameter $n$ there is an algorithm to construct all $g_{2}, g_{3}, B$ such that the group $M$ actually occurs. Here we give only an example of such a construction. We like to determine all algebraic Lamé equations with parameter $n=3 / 10$. According to Theorem 3.3 the monodromy group must be $G_{22}$. This has an invariant of degree 12 . Let $y_{1}(z), y_{2}(z)$ be two local solutions around infinity. Then there is a binary form $I$ of degree 12 such that $I\left(y_{1}, y_{2}\right)$ is invariant under monodromy. Hence it is a rational function in $z$. Moreover, since the local exponents at all finite points are non-negative, we have $I\left(y_{1}, y_{2}\right) \in \mathbb{C}[z]$. The explicit solutions read

$$
\begin{gathered}
y_{1}(z)=z^{3 / 20}\left(1+\frac{5 B}{4} \frac{1}{z}+\left(\frac{25 B^{2}}{192}-\frac{7 g_{2}}{1280}\right) \frac{1}{z^{2}}+\cdots\right), \\
y_{2}(z)=z^{-13 / 20}\left(1+\frac{5 B}{36} \frac{1}{z}+\left(\frac{25 B^{2}}{4032}+\frac{299 g_{2}}{8960}\right) \frac{1}{z^{2}}+\cdots\right) .
\end{gathered}
$$

The only degree twelve monomials that occur in $I\left(y_{1}, y_{2}\right)$ are therefore, $y_{1}^{11} y_{2}, y_{1}^{6} y_{2}^{6}, y_{1} y_{2}^{11}$. The others all contain fractional powers of $z$. We must find $\alpha, \beta$ such that $I=y_{1}^{11} y_{2}+$ $\alpha y_{1}^{6} y_{2}^{6}+\beta y_{1} y_{2}^{11} \in \mathbb{C}[z]$. Notice that the three relevant monomials are of order $-1,3,7$ in $1 / z$. Up to order $1 / z^{3}$ we have

$$
\begin{aligned}
I= & z+\frac{125 B}{9}+\frac{10000 B^{2}-3 g_{2}}{112} \frac{1}{z}+ \\
& +\frac{750000 B^{3}+650 B g_{2}-63 g_{3}}{2128} \frac{1}{z^{2}}+O\left(\frac{1}{z^{3}}\right) .
\end{aligned}
$$

The coefficients of $1 / z$ and $1 / z^{2}$ must be zero. Notice that through the substitution $z \rightarrow \lambda z$ in the Lamé equation the parameter $B$ changes into $B / \lambda$. Hence after suitable normalisation we can assume that $B$ has some arbitrarily given value. We take $B=$ $1 / 100$. It then follows from the vanishing of our two coefficients that $g_{2}=1 / 3$ and $g_{3}=5 / 108$. Applying Kovacic's algorithm to this particular case shows that we have indeed an algebraic differential equation.

5. Proof of Theorem 1.1. Given a linear differential equation from $\mathcal{A}_{0}$, let $M \subset$ $G L(2, \mathbb{C})$ be its finite Galois group. The conjugacy class of $M$ depends on the choice of a local basis $y_{1}, y_{2}$ with respect to which $M$ is determined. According to F.Klein's work, $y_{1}, y_{2}$ can be chosen in such a way that $M$ modulo scalars is one of a concrete list of possible groups in $\operatorname{PGL}(2, \mathbb{C})$. They are the cyclic group $C_{N}$ of order $N$, the dihedral group $D_{N}$ of order $2 N$, the tetrahedral group $A_{4}$, the octahedral group $S_{4}$ and the icosahedral group $A_{5}$. Let $G$ be such a group. A rational function $f(z)$ is called $G$ invariant when $f\left(\frac{a z+b}{c z+d}\right)=f(z)$ for every $\left(\begin{array}{ll}a & b \\ c & d\end{array}\right) \in G$. The $G$-invariant rational functions 
form a subfield of $\mathbb{C}(z)$ which we will denote by $\mathbb{C}(z)^{G}$. Klein constructed for each $G$ an explicit rational function $j_{G}(z) \in \mathbb{C}(z)$ such that $j_{G}$ generates $\mathbb{C}(z)^{G}$. Moreover, $j_{G}$ ramifies only above $0,1, \infty$.

Now consider the composite function $R(z)=j_{G}\left(y_{1} / y_{2}\right)$. Then $R(z)$ is invariant under monodromy, hence a meromorphic function on $\mathbb{P}^{1}$, i.e. $R(z) \in \mathbb{C}(z)$. Let $z_{0} \in \mathbb{P}^{1}$. The ramification order of $R(z)$ at $z_{0}$ is equal to the local exponent difference of $L y=0$ at $z_{0}$ times the ramification order of $j_{G}$ at $y_{1}\left(z_{0}\right) / y_{2}\left(z_{0}\right)$. This implies in particular that any point $z_{0}$ where the local exponent difference is not an integer, must be mapped to a ramification point of $j_{G}$ by $z_{0} \mapsto y_{1}\left(z_{0}\right) / y_{2}\left(z_{0}\right)$. Since $j_{G}$ ramifies only above $0,1, \infty$, we conclude that $R\left(z_{0}\right) \in\{0,1, \infty\}$. Let $z_{0}$ be any point such that $R\left(z_{0}\right) \neq 0,1, \infty$. Then $z_{0}$ must have integral exponent difference. Since our equation is pure this difference is 1 and therefore $R(z)$ is unramified in $z_{0}$. We conclude that $R(z)$ is a so-called Belyi function, a rational function $R: \mathbb{P}^{1} \rightarrow \mathbb{P}^{1}$ such that $R$ ramifies only above $0,1, \infty$.

According to [Schn, Lemma I.1] the set of Belyi functions is countable when we consider two Belyi functions $f(z), f\left(\frac{a z+b}{c z+d}\right)$ as equivalent. The set of functions $j_{G}$ is also countable and therefore the set of ratios $y_{1}(z) / y_{2}(z)$ modulo fractional linear transformations in $z$ is countable. Suppose now that two differential equations $\tilde{L} y=0$ and $L y=0$ give rise to the same quotient $y_{1} / y_{2}=\tilde{y}_{1} / \tilde{y}_{2}$. Differentiate both sides to get $W / y_{2}^{2}=\tilde{W} / \tilde{y}_{2}^{2}$ where $W$ and $\tilde{W}$ are the Wronskian determinants of the differential equations. For example $W(z)=y_{1}^{\prime} y_{2}-y_{1} y_{2}^{\prime}$. It is well-known that $W(z)=S(z)^{a}$ for some $S(z) \in \mathbb{C}(z)$ and $a \in \mathbb{Q}$. And similarly $\tilde{W}(z)=\tilde{S}(z)^{\tilde{a}}$. Hence $\tilde{y}_{2}=\tilde{S^{\tilde{a}} / 2} S(z)^{-a / 2} y_{2}$ and we conclude that $L y=0$ and $\tilde{L} y=0$ are equivalent. Hence, up to equivalence the set of equations in $\mathcal{A}_{0}$ is countable, as asserted.

\section{References}

[B] F. Baldassarri, On algebraic solutions of Lamé's differential equation, J. Differential Equations 41 (1981), 44-58.

[BW] F. Beukers and A. van der Waall, Lamé equations with algebraic solutions, preprint 1248, Dept. of Math., University of Utrecht.

[C] B. Chiarellotto, On Lamé operators which are pull-backs of hypergeometric ones, Trans. Amer. Math. Soc. 347 (1995), 2753-2780.

[K] F. Klein, Vorlesungen über das Ikosaeder, Teubner, Leipzig, 1884.

[Schn] L. Schnepps, Dessins d'enfants on the Riemann sphere, in: The Grothendieck Theory of Dessins d'Enfants, L. Schnepps (ed.), London Math. Soc. Lecture Note Ser. 200, Cambridge University Press, 1994, 48-77.

[Schw] H. A. Schwarz, Ueber diejenigen Fälle, in welchen die Gaussische hypergeometrische Reihe eine algebraische Function ihres vierten Elementes darstellt, J. Reine Angew. Math. 75 (1873), 292-335.

[ST] G. C. Shephard and J. A. Todd, Finite unitary reflection groups, Canad. J. Math. 6 (1954), 274-304.

[W] A. van der Waall, Lamé equations with finite monodromy, Ph.D. Thesis, University of Utrecht, 2002. 